\title{
Space gravity spectroscopy: the benefits of Taylor-Karman structured criterion matrices
}

\author{
P. Marinkovic, E. Grafarend, and T. Reubelt
}

Department of Geodesy and GeoInformatics, Stuttgart University, Geschwister-Scholl-Str. 24D, 70174 Stuttgart, Germany

\begin{abstract}
As soon as a space gravity spectroscopy was successfully performed, for instance by means of semicontinuous ephemeris of LEO - GPS tracked satellites, the problem of data validation appeared. It is for this purpose that a stochastic model for the homogeneous and isotropic analysis of measurements, obtained as "directly" measured values in LEO satellite missions (CHAMP, GRACE, GOCE), is studied. An isotropic analysis is represented by the homogeneous distribution of measured values and the statistical properties of the model are calculated. In particular, a correlation structure function is defined by the third order tensor (Taylor-Karman tensor) for the ensemble average of a set of incremental differences in measured components. Specifically, Taylor-Karman correlation tensor is calculated with the assumption that the analyzed random function is of a "potential type". The special class of homogeneous and isotropic correlation functions is introduced. Finally, a successful application of the concept is presented in the case study CHAMP and a comparison between modeled and estimated correlations is performed.
\end{abstract}

Key words. data validation, 3D correlation tensor, homogeneous and isotropic correlation functions, Taylor-Karman structure, CHAMP

\section{Introduction}

A significant problem of LEO satellites, both in geometry and gravity space, is the association of quality standards to Cartesian ephemeris in terms of variance-covariance matrix valued functions. As a pessimistic measure of the quality standards of LEO satellite ephemeris, a three-dimensional Taylor-Karman structured criterion matrix has been proposed, named in honor of Taylor (1938) and Karman (1938), the founders of the statistical theory of turbulence.

Correspondence to: P. Marinkovic

(marinkovic@gis.uni-stuttgart.de)
The concept of the Taylor-Karman criterion matrices was first introduced by Grafarend (1979) and subsequently further developed by Schaffrin and Grafarend (1982), Wimmer (1982), and Grafarend et al. $(1985,1986)$. With this contribution we extend the application of Taylor-Karman structured matrices to the third-dimension, namely to the long-arc orbit analysis.

If we assume the vector-valued stochastic process to be the gradient of a random scalar-valued potential, in particular its longitudinal and lateral correlation function or "the correlation function along-track and across-track", what would be the structure of a three-dimensional Taylor-Karman variancecovariance matrix? In order to answer this question, a threedimensional correlation-tensor and its decomposition in the case of homogeneity and isotropy is studied in Sect. 1, with the emphasis on $\mathbb{R}^{3}$. Additionally, we deal with a special class of homogeneous and isotropic tensor-valued correlation functions. They are derived, analyzed and applied to the data validation process. In Sect. 2, the theoretical concept for the application of the previously discussed criterion matrix in the geometric and gravitational analysis of LEO satellite ephemeris is presented. Finally, in Sect. 3, the case study CHAMP is used for the application of our theory concept followed by the results and conclusions.

\section{Homogeneous and isotropic variance-covariance ten- sor and correlation functions in a three-dimensional Euclidean space}

\subsection{Notions of homogeneity and isotropy}

The notions of homogeneity and isotropy for functions on $\mathbb{R}^{n}$ are briefly explained as following. The general context for these two definitions involves the action of a transitive group of motions on a homogeneous space and belongs to the extensive theory of Lie groups (Warner 1983, Yaglom 1987). However, it is important to clarify that the different notions of homogeneity and isotropy exist due to the vari- 


\section{Box 1.}

Tensor-valued correlation function of the second order

$$
\begin{aligned}
& \text { "two-point correlation function" } \\
& \Sigma\left(\mathbf{x}^{*}, \mathbf{x}^{* *}\right)=\sum_{i, j=1}^{3} \mathbf{e}^{i} \otimes \mathbf{e}^{j} \Sigma_{i j}\left(\mathbf{x}^{*}, \mathbf{x}^{* *}\right)=\sum_{i, j=1}^{3} \Sigma_{i j}\left(\mathbf{x}^{*}, \mathbf{x}^{* *}\right) \mathbf{e}^{i} \otimes \mathbf{e}^{j} \\
& r:=\left\|\mathbf{x}^{*}-\mathbf{x}^{* *}\right\| \quad \text { and } \quad \mathbf{r}:=\mathbf{x}^{*}-\mathbf{x}^{* *} \\
& \Sigma_{i j}(r)=\Sigma_{m}(r) \delta_{i j}+\left[\Sigma_{l}(r)-\Sigma_{m}(r)\right] \frac{\Delta x_{i} \Delta x_{j}}{r^{2}}, \quad i, j \in\{1,2,3\}
\end{aligned}
$$

"Cartesian versus spherical coordinates"$$
\Delta x_{1}=\Delta x:=\left(\mathbf{x}_{1}^{* *}-\mathbf{x}_{1}^{*}\right)=r \cos \beta \cos \alpha
$$$$
\Delta x_{2}=\Delta y:=\left(\mathbf{x}_{2}^{* *}-\mathbf{x}_{2}^{*}\right)=r \cos \beta \sin \alpha
$$$$
\Delta x_{3}=\Delta z:=\left(\mathbf{x}_{3}^{* *}-\mathbf{x}_{3}^{*}\right)=r \sin \beta
$$

"continuity condition of a potential type"

$$
\Sigma_{l}(r)=\frac{d\left[r \Sigma_{m}(r)\right]}{d r}=\Sigma_{m}(r)+r \frac{d \Sigma_{m}(r)}{d r}
$$
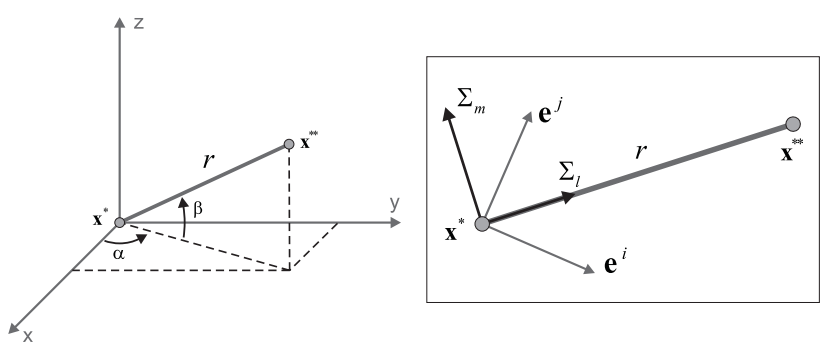

Fig. 1. Graphical representation of the longitudinal and lateral correlation functions.

ety of homogeneous spaces and transitive group actions on these spaces. The terminology introduced associates the notion of homogeneity and isotropy with the functions on $\mathbb{R}^{n}$ that are invariant under the translation group acting on $\mathbb{R}^{n}$. The notion of isotropy is defined for functions on $\mathbb{R}^{n}$ that are invariant under the orthogonal group acting on $\mathbb{R}^{n}$.

\subsection{Homogeneous and isotropic variance-covariance tensor (correlation tensor)}

Taylor (1938) proved that the homogeneous random field $X(\mathbf{t})$ correlation function $\Sigma(r)=\langle X(\mathbf{t}+\mathbf{r}) X(\mathbf{t})\rangle$ depends only on the length $r=\|\mathbf{r}\|$ of the vector $\mathbf{r}$ and not on its direction, where $\langle\ldots\rangle$ denotes the ensemble average. If the correlation function $\Sigma(r)$ of the homogeneous random field $X(\mathbf{t})$ in $\mathbb{R}^{n}$ has this property, then the field $X(\mathbf{t})$ is said to be an isotropic random field in $\mathbb{R}^{n}$. The corresponding cor-

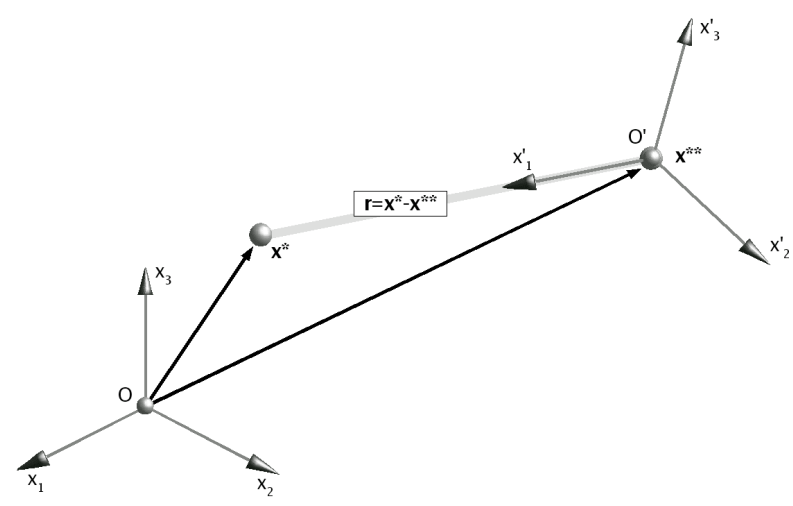

Fig. 2. Graphical representation of the correlation tensor transformation.

relation function $\Sigma(r)$ is then called an isotropic correlation function in $\mathbb{R}^{n}$ (or an $n$-dimensional isotropic correlation function). Processes, which satisfy the introduced postulates of homogeneity and isotropy, are said to be (widely) stationary (Yadrenko 1983). Note that for an isotropic random field in $\mathbb{R}^{n}$, all directions in space are obviously equivalent.

The decomposition of a homogeneous and isotropic variance-covariance tensor-valued function, shown in Box 1, was introduced by von Karman and Howarth (1938) by means of a more general and direct method than the one used by Taylor (1938). Additionally, Robertson (1940) refined and reviewed the Karman-Howarth equation in the light of a clas- 

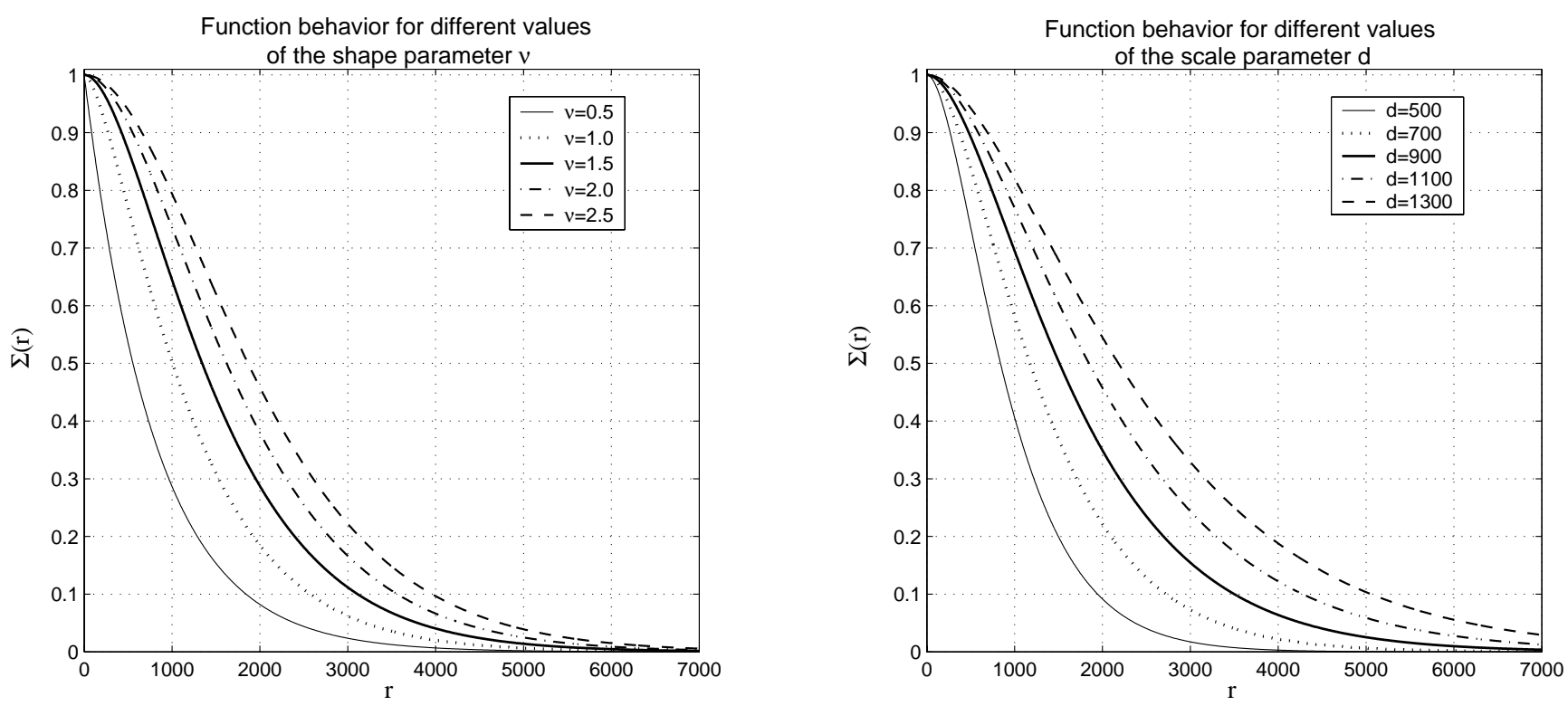

Fig. 3. The behavior of Tatarski's correlation function for different values of the shape parameter $v(d=900)$ and scale parameter $d$ $(v=3 / 2)$.

sical invariant tensor theory.

The decomposition of $\Sigma_{i j}\left(\left\|\mathbf{x}^{*}-\mathbf{x}^{* *}\right\|\right)$, with a special emphasis on $n=3$, is performed in terms of Cartesian coordinates and with respect to the orthonormal frame of reference $\left\{\mathbf{e}_{1}, \mathbf{e}_{2}, \mathbf{e}_{3} \mid 0\right\}$ attached to the origin 0 of a three-dimensional Euclidean space. $\left\|\mathbf{x}^{*}-\mathbf{x}^{* *}\right\|$ denotes the Euclidean distance $r$ between the two points $\mathbf{x}^{*}$ and $\mathbf{x}^{* *}$ of $\mathbb{E}^{3}:=\left\{\mathbb{R}^{3}, \delta_{i j}\right\}$. Longitudinal and lateral correlation functions, $\Sigma_{l}$ and $\Sigma_{m}$, are the structural elements of such a homogeneous and isotropic tensor-valued variance-covariance function which appear in the spherical tensor $\Sigma_{m}(r) \delta_{i j}$ as well as in the oriented tensor $\left[\Sigma_{l}(r)-\Sigma_{m}(r)\right] \Delta x_{i} \Delta x_{j} / r^{2}$ for all $i, j \in\{1,2,3\}$, see Eq. (2) and Fig. (1). $\delta_{i j}$ denotes the Kronecker or unit matrix, $\Delta x_{i}$ the Cartesian coordinate differences between the points $\mathbf{x}^{*}$ and $\mathbf{x}^{* *}$. These differences are also represented in terms of relative spherical coordinates $(\alpha, \beta, r)$, Eq. (3). Finally, the continuity condition of a potential type is formulated by Eq. (4), which provides the unique relation between the $\Sigma_{l}$ and $\Sigma_{m}$ (Taylor, 1938; Obuchow, 1958; Grafarend; 1979).

Due to its complexity, it is necessary to further elaborate on the previous equation set. The correlation tensor $\Sigma_{i j}(r)$ was transformed to a special coordinate system $O^{\prime} x_{1}^{\prime} x_{2}^{\prime} x_{2}^{\prime}$ in $\mathbb{R}^{3}$ instead of the initial set $O x_{1} x_{2} x_{3}$. The new set $O^{\prime} x_{1}^{\prime} x_{2}^{\prime} x_{3}^{\prime}$ is selected in such a way so that its origin $O^{\prime}$ is shifted by the vector $\mathbf{x}^{* *}$ with respect to the origin $O$, as illustrated in Fig. (2). This means that $O^{\prime}$ coincides with the terminal point of the vector $\mathbf{x}^{* *}$ that refers to the initial coordinates, while the axis $O^{\prime} x_{1}^{\prime}$ lies along the vector $\mathbf{x}^{*}-\mathbf{x}^{* *}$.

$\Sigma_{i j}^{\prime}(r)$, introduced here as explanatory functions, are the components of the correlation tensor $\Sigma_{i j}(r)$ in the new set of coordinates. The functions $\Sigma_{i j}^{\prime}(r)$ clearly depend only on the length $r=\|\mathbf{r}\|$ of the vector $\mathbf{r}$, since the direction of $\mathbf{r}$ is fixed in the new set of coordinates. In the space $\mathbb{R}^{3}$ exists a reflection which leaves the points $\mathbf{x}^{*}$ and $\mathbf{x}^{* *}\left(=O^{\prime}\right)$ unmoved and replaces the axis $O^{\prime} x_{j}^{\prime}$ by $-O^{\prime} x_{j}^{\prime}$, where $j \neq 1$ is a fixed number. However, it does not change the directions of all other coordinate axes $O^{\prime} x_{l}^{\prime}, l \in\{1,2,3\}$ and $l \neq j$. It follows that

$\Sigma_{i j}^{\prime}(r)=-\Sigma_{i j}^{\prime}(r)=0$ for $i \neq j$.

Hence, only the diagonal elements $\Sigma_{i i}^{\prime}(r)$ of $\Sigma_{i j}(r)$ can differ from zero. Further more, if $i \neq 1$ and $j \neq 1$, then the axis $O^{\prime} x_{i}^{\prime}$, by its rotation around the axis $O^{\prime} x_{j}^{\prime}$, can be transformed to the axis $O^{\prime} x_{1}^{\prime}$. Hence

$\Sigma_{22}^{\prime}(r)=\Sigma_{33}^{\prime}(r)$.

The tensors $\Sigma_{i j}^{\prime}$ and, consequently, $\Sigma_{i j}$ are symmetric and their components $\Sigma_{i j}^{\prime}(r)$ can take at most only two non-equal non-zero values at the already introduced longitudinal correlation function $\Sigma_{11}^{\prime}(r)=\Sigma_{l}(r)$ and the lateral correlation function $\Sigma_{22}^{\prime}(r)=\Sigma_{33}^{\prime}(r)=\Sigma_{m}(r)$, which specify in a unique way the correlation tensor. In order to obtain the explicit form for $\Sigma_{i j}(r)$, as the function of $\Sigma_{l}(r)$ and $\Sigma_{m}(r)$, the unit vectors of the old coordinate axes $O x_{1}, O x_{2}, O x_{3}$ along the axes of the new system $O^{\prime} x_{1}^{\prime}, O^{\prime} x_{2}^{\prime}, O^{\prime} x_{3}^{\prime}$ must be resolved and then $\Sigma_{i j}(r)$ can be represented as a linear combination of the functions $\Sigma_{k l}^{\prime}(r),(k \neq i, l \neq j$ and $k, l \in\{1,2,3\})$, which leads to Eqs. (1) and (2).

\subsection{Homogeneous and isotropic correlation functions}

It was shown in the previous section that for a homogeneous and isotropic random field defined on the Euclidean space $\mathbb{R}^{n}$, the correlation between $\mathbf{x}^{*}$ and $\mathbf{x}^{* *}$ depends only on the 


\section{Box 2.}

Isotropic correlation function and Schoenberg's characterization

"homogeneous and isotropic correlation function"

$$
\begin{gathered}
\Sigma\left(\mathbf{x}^{*}, \mathbf{x}^{* *}\right)=\Sigma\left(\left\|\mathbf{x}^{*}-\mathbf{x}^{* *}\right\|\right), \quad \mathbf{x}^{*}, \mathbf{x}^{* *} \in \mathbb{R}^{n} \\
r:=\left\|\mathbf{x}^{*}-\mathbf{x}^{* *}\right\| \\
\text { "the characterization of the } \Phi_{n} \text { class" } \\
\Sigma_{n}(r)=\int_{[0, \infty)} \Omega_{n}(r \nu) d W(\nu) \\
\Omega_{n}(r)=\Gamma(n / 2)\left(\frac{2}{r}\right)^{(n-2) / 2} J_{(n-2) / 2}(r) \\
" r e d u c t i o n \text { of the } \Omega_{n} \text { for } n=2 \text { and } n=3 " \\
\Omega_{2}(r)=J_{0}(r) \quad \text { and } \quad \Omega_{3}(r)=r^{-1} \sin r
\end{gathered}
$$

\section{Box 3.}

$$
\begin{gathered}
\text { Special classes of correlation functions } \\
\text { "Tatarski's class" } \\
\Sigma_{[\nu]}(r)=\frac{2^{1-\nu}}{\Gamma(\nu)}\left(\frac{r}{d}\right)^{\nu} K_{\nu}\left(\frac{r}{d}\right) \\
\text { "Shkarofsky's class" } \\
\Sigma_{[\nu, \delta]}(r)=\frac{\left(\frac{r^{2}}{d^{2}}+\delta^{2}\right)^{\nu / 2} K_{\nu}\left(\left(\frac{r^{2}}{d^{2}}+\delta^{2}\right)^{1 / 2}\right)}{\delta^{\nu} K_{\nu}(\delta)}
\end{gathered}
$$

Euclidean distance $\left\|\mathbf{x}^{*}-\mathbf{x}^{* *}\right\|$. Therefore, as shown in Box 2, we can distinguish a homogeneous and isotropic correlation function on $\mathbb{R}^{n}$ with the real-valued function $\Sigma(r)$ defined on $[0, \infty)$ and we denote by $\Phi_{n}$ the class of all continuous permissible functions $\Sigma(r) . \Phi_{n}$ is the class of all continuous functions $\Sigma(r):[0, \infty) \rightarrow$ such that $\Sigma(0)=1$ (we are working in terms of correlation not covariance) and the symmetric function $\Sigma(\|\cdot\|)$ is a positive definite on $\mathbb{R}^{n}$. The characterization of the classes $\Phi_{n}$, also shown in Box 2, is a well-known result of Schoenberg (1938). The function $\Sigma(r):[0, \infty) \rightarrow \mathbb{R}$ is an element of $\Phi_{n}$ if and only if it admits a representation in the form of Eq. (8), where $W$ is a probability measure on $[0, \infty), J_{(n-2) / 2}$ is the Bessel function of the first kind of order $(n-2) / 2$ and $\Gamma$ stands for the Gamma function. Hence, in for geodesy relevant spaces $\mathbb{R}^{2}$ and $\mathbb{R}^{3}$, Eq. (9) reduces to the form presented in Eqs. (10).
2.4 Tatarski's class of homogeneous and isotropic correlation functions

Many analytical candidate models for $\Sigma$ have been suggested in the literature (for example, Buell, 1972; Haslett, 1989), but we refer to Tatarski (1961) as being the first who elaborated on such correlation functions which fulfill all the conditions presented in the previous section. The Tatarski's correlation function class is shown in Box 3 and illustrated by Fig. 3. In addition to Tatarski's class, a very general family of correlation function models due to Shkarofsky (1968) is introduced, that came as the generalization of Tatarski's correlation function family. These two classes, which have been proved to be the members of $\Phi_{3}$ (Shkarofsky, 1968 and of $\Phi_{\infty}$ classes; Gneiting et al., 1999), can be applied to many geodetic problems, (e.g. Grafarend, 1979; Meier, 1981; Wimmer, 1982; Grafarend, 1985). 
In equation Box $3, K_{v}$ stands for a modified Bessel function of order $v, d>0$ is a scale parameter, and $\delta>0$ and $v$ are shape parameters. In the case of $\delta=0$ Shkarofsky's class reduces to Tatarski's class.

The special case of Tatarski's class appears if the shape parameter $v$ is the sum of a non-negative integer $k$ and $1 / 2$. Then the right-hand side of the equation can be written as a product of $\exp (-r / d)$ and a polynomial of degree $k$ in $r / d$ (e.g. Gneiting, 1999). In particular, in the case of $n=3$ dimensional Markov process of the $p=1$ order, shown in Fig. 4, the shape parameter is expressed as $v=(2 p+1) /(n-1)=3 / 2$ and results in the following simplification of Eq. (11):

$\Sigma_{[3 / 2]}(r)=\left(1+\frac{r}{d}\right) \exp \left(-\frac{r}{d}\right)$.

\section{Three-dimensional Taylor-Karman criterion matrix in the geometric and gravitational analysis of LEO satellite ephemeris}

We have so far analyzed the theoretical background and solution for design of the homogeneous and isotropic TaylorKarman correlation tensor. The question is, how this theoretical concept applies to the geometric and gravitational analysis of LEO satellite ephemeris.

The basic idea is, that the errors in position vectors of LEO satellites constitute a vector-valued stochastic process. Following this concept, a satellite orbit of LEO - GPS tracked satellites is an inhomogeneous and anisotropic field of error vectors and the error situation is described by the covariance function. As it is well known, the error situation of a newly determined position in a three-dimensional Euclidean space is the best, when the error ellipsoid is a sphere (isotropy) with a minimal radius and if the error situation is uniform over the complete satellite orbit (homogeneity). This "ideal" situation can be explained by the three-dimensional TaylorKarman structured criterion matrix of Baarda-Grafarend (potential) type. Then the correlations between the vectors of pseudo-observations of satellite ephemeris are described by the longitudinal and lateral correlation functions.

The characteristic correlation functions can be estimated by matching the correlation tensor with a three-dimensional Markov process of the 1st order and with the introduction of some additional information about the underlying process. The correlation analysis is performed with the assumption that the vector valued three-dimensional random function is of "potential type" (Grafarend, 1979), i.e. is the gradient of a random scalar function "signal $s(\mathbf{x})$ ". The structure of the random function $s(\mathbf{x})$ is outlined as a $n$-dimensional Markov process of the $p$-th order. Figure 4 illustrates the case $n=3$ and $p=1$.

One of the simplest differential equation of such a process has the form given by

$\left(\nabla^{2}-\alpha^{2}\right)^{p} s(\mathbf{x})=e(\mathbf{x})$

where $e(\mathbf{x})$ is a white noise. If the Laplace operator can be applied to the homogeneous random scalar function, then it

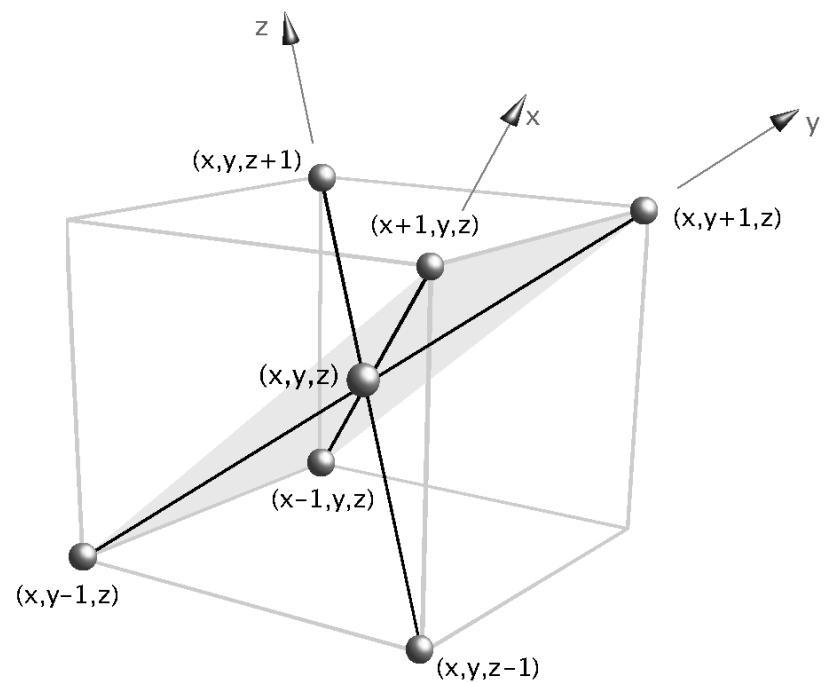

Fig. 4. The six point interaction in the grid; the three-dimensional Markov process of the 1st order (autoregressive process); the gray rectangle represents the same process in two-dimensions.

transforms this function into a new homogeneous random function, having the spectral density that corresponds to the spectral density of the correlation function of Eqs. (11) and (12), see Whittle (1954), Heine (1955) and Whittle (1963). Hence the homogeneous solution of Eq. (14) (if it exists) must have the spectral density that corresponds to the spectral density of the correlation function.

Box 4 summarizes the representation of the homogeneous and isotropic correlation functions of type (i) signal correlation function $\Sigma$, (ii) longitudinal correlation function $\Sigma_{l}$ and (iii) lateral correlation function $\Sigma_{m}$.

\section{Results and conclusions}

\subsection{Case study: CHAMP}

As the numerical test in this study, we processed two data sets: the two three-dimensional $\left\{x\left(t_{k}\right), y\left(t_{k}\right), z\left(t_{k}\right)\right\}$ and $\left\{x\left(t_{d}\right), y\left(t_{d}\right), z\left(t_{d}\right)\right\}$ Cartesian ephemeris time-series of CHAMP satellite orbit for the test period from the day 140 to 150 of 2001 (20 May to 30 May, both inclusive). We analyzed in total 27360 triples of satellite positions. Both time series are indexed with a 30 second sampling rate and referenced to a kinematic (index $k$ ) and a dynamic CHAMP orbit (index $d$ ). The dynamic orbit, used as a reference, provides us with ephemeris differences between the two orbits. The estimation of ("real") auto and cross correlations between the vectors of pseudo-observations as functions of time, can be performed as in Priestley (1981).

According to Box 1 and Box 4 the Taylor-Karman structured ("ideal") correlations are computed from the threedimensional $\left\{x\left(t_{k}\right), y\left(t_{k}\right), z\left(t_{k}\right)\right\}$ time series. The adopted scale parameter is $d=(2 / 3) R_{\text {char }}$, where $R_{\text {char }}$ is the char- 
Box 4.

Longitudinal and lateral correlation function for a homogeneous and isotropic

vector-valued random field of potential type, 1st order Markov process

"condition for a process of potential type"

$$
\begin{gathered}
\Sigma(x) \longrightarrow \frac{2 p}{r^{2}} \int_{0}^{r} x \Sigma(x) d x \longrightarrow \\
\longrightarrow \Sigma_{l}(r)=\frac{d}{d r}\left[r \Sigma_{m}(r)\right] \\
\text { "input" } \\
\Sigma(r)=\frac{2^{-1 / 2}}{\Gamma(3 / 2)}\left(\frac{r}{d}\right)^{3 / 2} K_{3 / 2}\left(\frac{r}{d}\right)=\left(1+\frac{r}{d}\right) \exp \left(-\frac{r}{d}\right) \\
\text { "output" } \\
\Sigma_{l}(r)=-6\left(\frac{r}{d}\right)^{-2}+\exp \left(-\frac{r}{d}\right)\left[4+2\left(\frac{r}{d}\right)+6\left(\frac{r}{d}\right)^{-1}+6\left(\frac{r}{d}\right)^{-2}\right] \\
\Sigma_{m}(r)=6\left(\frac{r}{d}\right)^{-2}-\exp \left(-\frac{r}{d}\right)\left[2+6\left(\frac{r}{d}\right)^{-1}+6\left(\frac{r}{d}\right)^{-2}\right]
\end{gathered}
$$
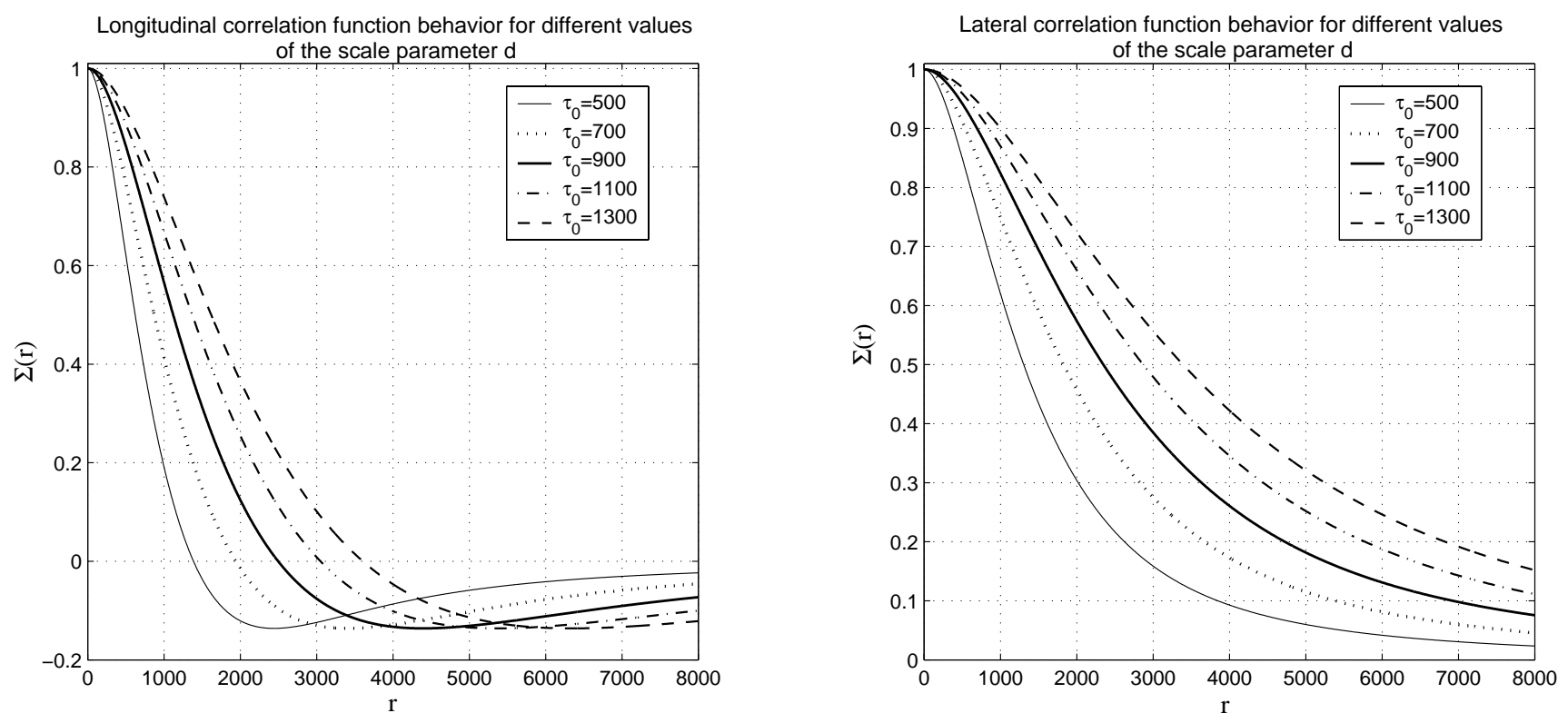

Fig. 5. The behavior of the longitudinal and lateral correlation functions, Eqs. (15) and (16), for different values of the scale parameter $d$ $(v=3 / 2)$.

acteristic length of the process. The characteristic length is defined by the arc length of 2400 seconds ( 80 points for the 30 seconds sampling rate). The both parameters are experimentally estimated. For further details on the scale parameter and characteristic length, please see Wimmer (1982).

The numerical results of the study are graphically presented in Fig. 6. The gray area represents the estimated ("real") correlation situation along the satellite arc as presupposed by Austen et al. (2001). The high auto and low cross correlations between CHAMP satellite positions for approximately $20 \mathrm{~min}$ of an orbit arc are very evident. The TaylorKarman structured correlation (black line), as theoretically assumed, gives an upper bound of the "real" correlation situation along the satellite orbit. 


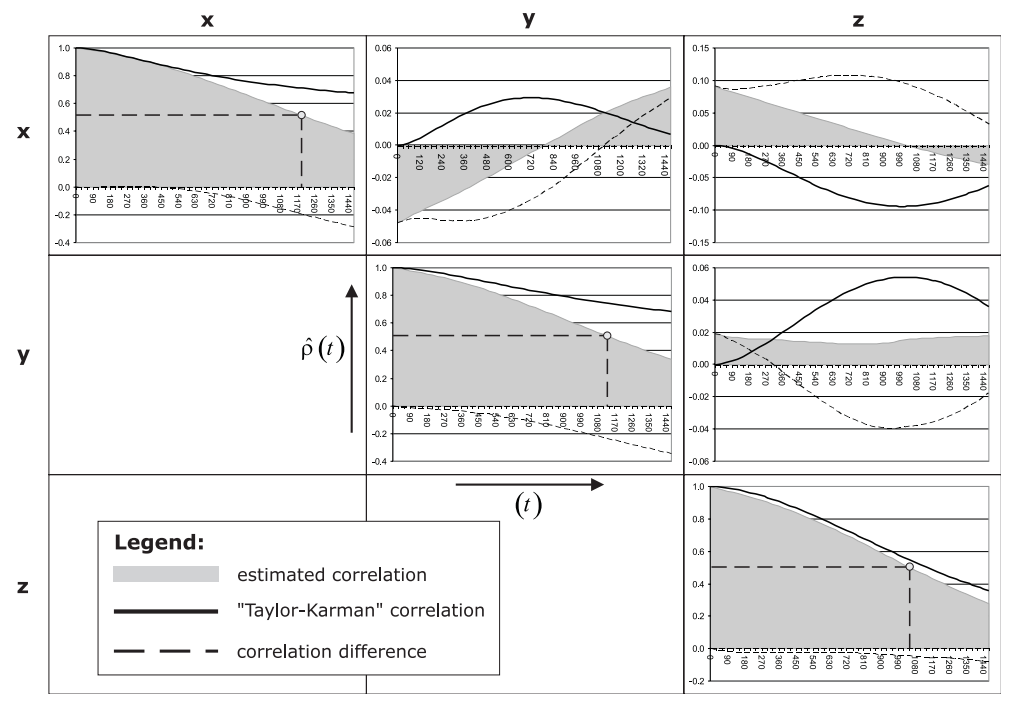

Fig. 6. Graphical representation of the numerical study results ( 20 min correlation length, 40 points for the 30 s sampling rate).

\subsection{Concluding remarks}

With this study, the statistical analysis of Grafarend (1979) is successfully extended to the third dimension and applied into a homogeneous and isotropic stochastic analysis of semi-continuous ephemeris of LEO - GPS tracked satellites. Tatarski's correlation function class is also introduced.

In order to obtain the "ideal" error information along the satellite orbit, a derived mathematical model for TaylorKarman correlation tensor was applied. The model was developed by matching the correlation tensor with a random function outlined as a three-dimensional Markov process of the 1st order. The characteristic functions of the tensor, longitudinal and lateral components, are derived with the assumption that the random function is of "potential type". Further on, under the continuity condition, exists the unique relation between the characteristic functions. The implemented random function is a member of Tatarski's correlation function class.

Behind the Taylor-Karman correlation tensor lies the basic idea of the data validation process and necessity to determine the quality of the underlying process (geodetic network, satellite orbit, etc.). With this concept, canonical comparison of "real" and "ideal" covariance matrix is performed and, instead of referring to the quality of the process through one number, the whole spectrum of information about the process quality becomes available.

In the light of the new satellite missions (CHAMP, GRACE, GOCE), there are further applications of TaylorKarman tensor in weighting schemes, in regularization algorithms, etc., of the LEO satellites measurements. The new applications are enabled: (i) with the introduction of a higher order Markov process and/or different shape and scale parameters, (ii) by matching the longitudinal and lateral components of the tensor with the experimental results, and (iii) with the higher order correlation tensor modeling. These will be the subject of future articles.
Acknowledgements. We thank Professor M. Rothacher and his group at the Institute of Astronomical and Physical Geodesy, TU Munich, for providing us the CHAMP kinematic orbit for the test period. This study was also supported by the German Federal Ministry of Education and Research under grant number 03F0333C. This support is gratefully acknowledged.

\section{References}

Austen, G., Grafarend, E. W., and Reubelt, T.: Analysis of the Earth's gravitational field from semi continuous ephemeris of a low earth orbiting GPS-tracked satellite of type CHAMP, Grace or Goce, in: Vistas for Geodesy in the New Millennium, (Eds) Ádám and Schwarz, International Association of Geodesy Symposia 125, 309-315, 2001.

Baarda, W.: S-Transformations and criterion matrices. Netherlands Geodetic Commission Volume 7, No. 1, 1973.

Batchelor, G. K.: The theory of homogeneous turbulence, Cambridge University Press, Cambridge, 1953.

Buell, C. E.: Correlation functions for wind and geopotential on isobaric surfaces, Journal of Applied Meteorology, 11, 51-59, 1972.

Comte, F. and Rozenholc, Y.: Adaptive estimation of mean and volatility functions in (auto-) regressive models, Stochastic Processes and their Applications 97, 111-145, 2002.

Gaspari, G. and Cohn, S. E.: Construction of correlation functions in two and three dimensions, Q. J. R. Meteorol. Soc., 125, 723757, 1999.

Grafarend, E. W. and Schaffrin, B.: Kriterion-Matrizen I - zweidimensionale homogene und isotrope Netze, ZfV, 4, 133-149, 1979.

Grafarend, E. W.: Criterion matrices for deforming networks, in: Optimization and Design of Geodetic Networks, (Eds) Grafarend, E. W. and Sanso, F., Springer Verlag, 363-428, 1985.

Grafarend, E. W., Krumm, F., and Schaffrin. B.: Criterion matrices of heterogeneously observed three-dimensional networks, Manuscripta Geodaetica, 10, 3-22, 1985.

Grafarend, E. W., Krumm, F., and Schaffrin. B.: Kriterion-Matrizen III - zweidimensionale homogene und isotrope Netze, ZfV, 5, 197-207, 1986. 
Gneiting, T.: Correlation functions for atmospheric data analysis, Q. J. R. Meteorol. Soc., 125, 2449-2464, 1999.

Gneiting, T. and Sasvári, Z.: The characterization problem for isotropic covariance functions, Q. J. R. Meteorol. Soc., 125, 2449-2464, 1999.

Haslett, J.: Space time modeling in meteorology - a review, Bull. Int. Stat. Inst., 53, 229-246, 1989.

Heine, V.: Models for two-dimensional stochastic process, Biometrika, 42, 170-178, 1955.

Kármán, T. and Howarth, L.: On the statistical theory of turbulence, Proc. Roy. Soc., A-164, 192-215, 1938.

Lai, T. L. and Lee, C. P.: Information and prediction criteria for model selection in stochastic regression and ARMA models, Statistica Sinica, 7, 285-309, 1997.

Meier, S.: Planar geodetic covariance functions, Rev. of Geophysics and Space Physics, 19, 673-686, 1981.

Obuchow, A. M.: Statistische Beschreibung stetiger Felder, in: Sammelband zur statistischen Theorie der Turbulenz, Goering H. (hrsg.), Berlin, 1958.

Priestley, M. B.: Spectral analysis and time series: Vols. 1 and 2. Academic Press, London, 1981.

Robertson, H. P.: The invariant theory of isotropic turbulence, Proc. Camb. Phil. Soc., 36, 209-223, 1940.

Schaffrin, B. and Grafarend, E. W.: Kriterion-Matrizen II - zweidimensionale homogene und isotrope Netze, ZfV, 107, 485-483, 1982.
Schoenberg, I. J.: Matric spaces and completely monotone functions, Annals of Mathematics, 39, 811-841, 1938.

Shkarofsky, I. P.: Generalized turbulence and space correlation and wave-number spectrum-function pairs, Canadian Journal of Physics, 46, 2133-2153, 1968.

Tatarski, V. I.: Wave propagation in a turbulent medium, McGrawHill Book Company, New York, 1961.

Taylor, G. I.: The spectrum of turbulence, Proc. Roy. Soc. A-164, 476-490, 1938

Temme, N. M.: Special functions: An introduction to the classical functions of mathematical reference, John Wiley and Sons, Inc. New York, 1996.

Warner, F. W.: Foundations of differentiable manifolds and Lie groups, Springer-Verlag, New York, 1983.

Whittle, P.: On stationary process in the plane, Biometrika 41, 434 449, 1954

Whittle, P.: Stochastic processes in several dimensions, Bull. Int. Statist. Inst., 40, 974-994, 1963.

Wimmer, H.: Ein Beitrag zur Gewichtsoptimierung geodaeticher Netze, Report C269 - Deutsche Geodaetische Komission, Bayerische Akademie der Wissenschaften, München, 1982.

Yadrenko, M. I.: Spectral theory of random fields, Optimization Software: Publications Division, New York, 1983.

Yaglom, A. M.: Correlation theory of stationary and related random functions, Vol. I: Basic Results, Springer-Verlag, New York, 1987. 